\title{
Quantum Correlation Calculation Via Semiclassical Concept Using Matlab Model
}

\author{
Khalid Mohammed Haroun ${ }^{1,4}$, Ahmed Mohammed Gomaa ${ }^{2}$, Sara IdrisBabiker ${ }^{3}$ \\ ${ }^{1}$ Department of Physics, Faculty of Radiobiological Sciences \& Medical Imaging, Al-Zaiem Al-Azhari University, Khartoum, Sudan \\ ${ }^{2}$ Institue of Laser- Sudan University of Science \& Technology, Khartoum, Sudan \\ ${ }^{3}$ Faculty of Science, Al-Baha University, AL-Baha, Kingdom of Saudi Arabia \\ ${ }^{4}$ Department of Basic Sciences, Gezira College of Technology, Khartoum, Sudan
}

Email address:

agumaa2@gmail.com (A. M. Gomaa)

To cite this article:

Khalid Mohammed Haroun, Ahmed Mohammed Gomaa, Sara Idris Babiker. Quantum Correlation Calculation Via Semiclassical Concept Using Matlab Model. Mathematical Modelling and Applications. Vol. 3, No. 3, 2018, pp. 39-43. doi: 10.11648/j.mma.20180303.11

Received: October 10, 2018; Accepted: October 25, 2018; Published: December 26, 2018

\begin{abstract}
Entanglement is a phenomenon where two particles are linked by some kind of particularity. An electron can be entangled by its spin. Photons can be entangled through its polarization. In this worka made of simple modeling program with simple representing of polarizing beam splitter using quantum probability law, statistic correlation and entanglement argument is established. The work aimed to simulate Eienstien Poldosky Rosen Bom Gedanken experiment using Matlab, and to calculate the Bell's inequality. The obtained results of correlation were above ' 2 ' in many trials that considered an obvious violation of classic Bell's inequality value. But the values were not stable, and sometimes the values down to classic value of correlation(less than '2'). However the results obtained at many trials shows obvious indicator of Bell's inequality maximum classic value ' 2 ' violation, that by modeling the probability amplitude in Polarizing beam splitter just as Malus's law of intensity.
\end{abstract}

Keywords: Bell's Inequality, Correlation, Estimation, Violation

\section{Introduction}

One of the most mysterious of quantum mechanics is the entanglement phenomenon, which shows strong correlation of the behavior of quantum objects, even when they are separated by a large distance $[1,2]$. According to quantum mechanics, a state of each such an object cannot be described independently, instead a quantum state must be described for the system as a whole [3]. By other words entangled state cannot be factorized into a product of two states associated to each object. One of way to obtain an entangled photons is by spontaneous parametric down conversion (SPDC). A 100mW ion-argon pump laser beam is incident on a pair of orthogonally polarized type I Beta Barium Borate crystals. As the pump beam interacts with these nonlinear crystals, single photon split into entangled "signal" and "idler" photons with wavelengths longer than the pump [4]. The entanglement phenomenon was considered for the first time by A. Einstein, B. Podolsky, and N. Rosen and was developed further by D. Bohm who described what came to be known as the EPRB Gedanken experiment and EPRB paradox [3].

The first quantitative criterion which describes such a paradox was proposed by J. Bell (Bell's inequality). The Bell's inequality, derived on the basis of the local hiddenvariable theories. It is considered that an experiment in which the violation of the Bell's inequality occurs cannot be explained based on the local realism view. Bell's inequality gave the tool for experimental verification of the counterintuitive predictions of quantum mechanics [5]. Later, J. F. Clauser, M. A. Horne, A. Shimony and R. A. Holt (CHSH) proposed a new criterion and an experiment to test the local hidden-variable theories.

\section{Gedanken Experiment Description}

The optical version of Gedanken experiment of Einstein, Podolsky and Rosen, in which a source S emits entangled 
pairs of photons with different frequencies, $v_{1}$ and $v_{2}$, which propagate along opposite directions $\pm \mathrm{Z}$.

Let's suppose that the state entangled which describes polarization of the two photons given by:

$$
|\varphi\rangle=\frac{1}{\sqrt{2}}(|00\rangle+|11\rangle)
$$

where $|0\rangle$ and $|1\rangle$ represent states describing two different orthogonal directions of linear polarization. Two polarizing beam splitters 1 and 2, placed at the sides of the source and oriented in the directions given by unit vectors a and $b$, have the aim to analyze photons and see their tracks [6]. So it is possible to make measurements on linear polarization of two photons through the analysis of two detectors placed next to each of the polarizing beam splitters (PBSs). Both will give result +1 or -1 depending on whether the photon polarization occurs in a direction parallel or perpendicular to the one of the PBS itself.

Let $\mathrm{P}_{ \pm}(\mathrm{a})$ represents the probability of obtaining the result \pm 1 for $v_{1}$, and $\mathrm{P}_{ \pm}$(b) that one of obtaining \pm 1 for $v_{2}$, for these measurements quantum mechanical predictions for single detection are:

$$
\begin{gathered}
P_{+}(a)=P_{-}(a)=1 / 2 \\
P_{+}(b)=P_{-}(b)=1 / 2
\end{gathered}
$$

According to the fact that no polarization can be established for single photon; therefore any measurement will give a random result.

Defining now $\mathrm{P}_{ \pm}(\mathrm{a}, \mathrm{b})$ to be the probability of combined detection of $v_{1}$, in channel \pm of $\mathrm{a}$, and $v_{2}$, in channel \pm of $\mathrm{b}$, quantum mechanical prediction for combined detection are:

$$
\begin{gathered}
P_{++}(a, b)=P_{-}(a, b)=\frac{1}{2} \cos ^{2}(a, b) \\
P_{ \pm-}(a, b)=P_{-+}(a, b)=\frac{1}{2} \sin ^{2}(a, b)
\end{gathered}
$$

Where $(a, b)$ is the angle between $a$ and $b$.

In particular, in the case for $(a, b)=0$, the equations( 3 ) become:

$$
\begin{gathered}
P_{++}(a, b)=P_{-}(a, b)=1 \\
P_{ \pm-}(a, b)=P_{\mp}(a, b)
\end{gathered}
$$

that is, whether the photon $v_{1}$ gives result +1 (whose probability is $50 \%$ ), then photon $v_{2}$ as well will give certainly result +1 (and that also for the result-1), that means total correlation.

Carrying out the experiments for four different orientations $a$, a and $b, b^{\prime}$ of the polarizer a and b, one can calculate the estimation parameter $\mathrm{S}$ :

$$
S=E(a, b)-E\left(a, b^{\prime}\right)+\mathrm{E}\left(a^{\prime}, b\right)+E\left(a^{\prime}, b^{\prime}\right)
$$

This correlation coefficient provides a quantitative criterion in order to quantify the correlation between random results obtained from any independent individual measurement.

The CHSH inequality in concept of hidden variable predicts that:

$$
-2 \leq S_{H V} \leq 2
$$

Where $\mathrm{S}_{\mathrm{HV}}$ is the correlation value calculated from hidden variable theory.

By using specific set of angles, such as:

$$
(a, b)=\left(a^{\prime}, b\right)=\left(a^{\prime}, b^{\prime}\right)=22.5^{\circ} \text { and }\left(a, b^{\prime}\right)=67.5^{\circ}
$$

and if we calculate CHSH value using quantum mechanics the predicted value is:

$$
S_{Q M}=2 \sqrt{2}
$$

This means that the correlation value in quantum objects can be more than 2 some cases, and this can show a new remote affection idea in physics [7].

$\mathrm{CHSH}$ inequality was testable in numerous experiments, all of which have shown agreement with quantum mechanics rather than the principle of local realism $[2,8]$.

So that quantum mechanical calculations suggest that although each individual measurement gives random results, these random results are correlated. This means that immediately after the first measurement, photon $v_{1}$ takes the polarization $|\mathrm{a}\rangle$, the distant photon $v_{2}$ which has not yet interacted with any polarizer has also been projected into the state $|a\rangle$ with a well define polarization, parallel to the one found for photon $v_{1}$ [3].

\section{Modeling Program}

To make our model, random generator function used to play the role of entangled photons source to emit two photons $v_{1}$ and $v_{2}$, at same polarization. The function generates zeroes and ones randomly, where " 0 " represent a horizontal polarization, and " 1 " represent a vertical one. Two photons has been emit at the same time and in the same polarization but in opposite direction. Then two observers Alice and Bob measure the polarization of two photons $v_{1}$ and $v_{2}$ respectively, randomly and independently.

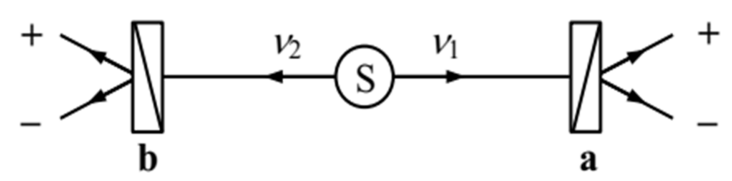

Figure 1. Entanglement source, Alice and Bob random measurements

Alice choose randomly an angle a from $\left\{0, \frac{\pi}{8}, \frac{\pi}{4}\right\}$ to measure the polarization of photon $v_{1}$. And the program used random choice function to simulate Alice options [9]. the model simulate the measurement results of photons polarization after passing PBS, by considering the probability of photon to pass through vertical and horizontal directions $\operatorname{are}^{2} \sin ^{2}(a)$ and $\cos ^{2}(a)$, respectively:

If $\cos ^{2}(a)>\sin ^{2}(a)$ and the incident photon was at "0" state, Alice will obtain ' +1 ', and $\operatorname{ifsin}^{2}(a)>\cos ^{2}($ a)Alice will get ' -1 ' result. And if $\cos ^{2}(a)=\sin ^{2}(a)$, Alice will get' +1 ' or 
'-1' randomly. Here we used the $\cos ^{2}(a)$ and $\sin ^{2}(a)$ to represent the probability in quantum mechanics, this is analogous to Malus intensity law [10]. the random results when $\cos ^{2}(a)=\sin ^{2}(a)$ must ruled by quantum amplitude (the results must be half' +1 ' and half'-1'), but the model chose it as classic randomization, to see if one can obtain violation of classical correlation or not.

For Bob measurement, the model chose randomly angle from $b=\left\{-\frac{\pi}{8}, 0, \frac{\pi}{8}\right\}$, and did same as Alice but independently.

By using $\frac{\pi}{8}$ trials when Alice and Bob chose a different polarization angles like: $\left(0, \frac{\pi}{8}\right),\left(0,-\frac{\pi}{8}\right),\left(\frac{\pi}{4}, \frac{\pi}{8}\right),\left(\frac{\pi}{4},-\right.$ $\frac{\pi}{8}$ ) as reported by $\mathrm{CHSH}$, the model calculated the correlation Estimation "E" for many pairs of measurement results, where:

$$
E=\frac{P_{++}(a, b)+P_{-}(a, b)-P_{ \pm}(a, b)-P_{\mp}(a, b)}{P_{++}(a, b)+P_{-}(a, b)+P_{ \pm}(a, b)+P_{\mp}(a, b)}
$$

Then the model calculated the value(S) of CHSH Bell's inequality where:

$$
\mathrm{S}=\mathrm{E}_{1}+\mathrm{E}_{2}-\mathrm{E}_{3}+\mathrm{E}_{4}
$$

Where, $\mathrm{E}_{1}\left(0, \frac{\pi}{8}\right), \mathrm{E}_{2}\left(0,-\frac{\pi}{8}\right), \mathrm{E}_{4}\left(\frac{\pi}{4}, \frac{\pi}{8}\right), \mathrm{E}_{3}\left(\frac{\pi}{4},-\frac{\pi}{8}\right)$

The model, used 50 photons pairs firstly and ran the program ten times, and then chose a ten results which had sequences values above "2". Then by exceeding the number of pairs with 50 , the program executed up to 500 .

Figure 2 shows the flow chart of the modeling.

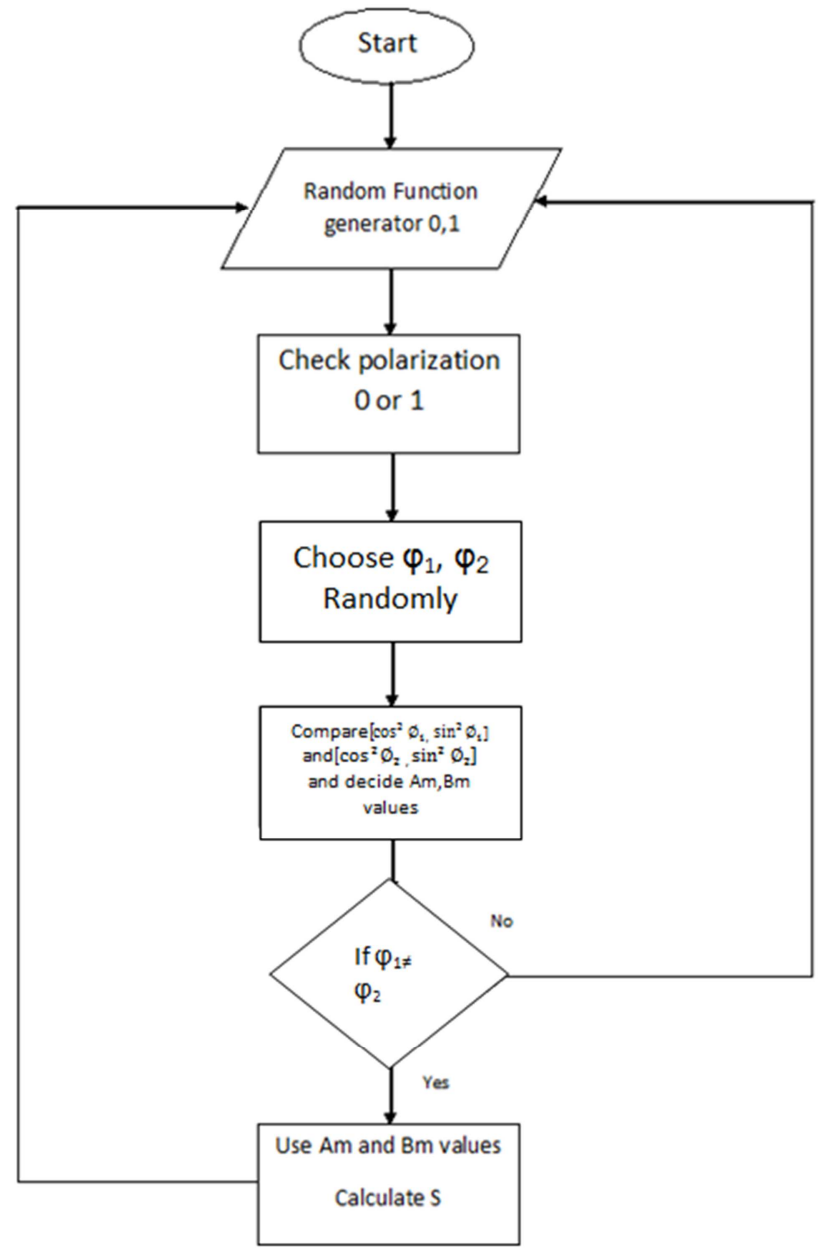

Figure 2. Modeling flow chart.

\section{Results and Discussion}

The obtained results from the modeling of entangled photon pairs are represented in the table 1:

\begin{tabular}{|c|c|c|c|c|c|c|c|c|c|c|}
\hline $\mathbf{N}$ & 50 & 100 & 150 & 200 & 250 & 300 & 350 & 400 & 450 & 500 \\
\hline S & 2.60 & 2.50 & 2.33 & 2.340 & 2.43 & 2.08 & 2.22 & 2.25 & 2.27 & 2.35 \\
\hline " & 2.29 & 1.89 & 2.38 & 2.39 & 2.17 & 2.0 & 2.20 & 2.14 & 2.18 & 2.30 \\
\hline " & 2 & 2.10 & 2.41 & 2.17 & 2.11 & 2.04 & 2.15 & 2.18 & 2.16 & 2.37 \\
\hline " & 2.17 & 2.09 & 2.40 & 2.17 & 2 & 2.04 & 2.11 & 2.10 & 2.14 & 2.28 \\
\hline$"$ & 2.24 & 2.10 & 2.34 & 2.09 & 2.08 & 2.08 & 2.17 & 2.08 & 2.10 & 2.14 \\
\hline " & 2.09 & 2.12 & 2.39 & 2.07 & 2.10 & 2.11 & 2.06 & 2.12 & 2.0 & 2.17 \\
\hline$"$ & 2.17 & 2.09 & 2.26 & 2.06 & 2.05 & 2.06 & 2.08 & 2.13 & 2.04 & 2.19 \\
\hline " & 2.08 & 2.12 & 2.17 & 2.05 & 2.04 & 2.02 & 2.12 & 2.08 & 2.02 & 2.19 \\
\hline " & 2.15 & 2.15 & 2.15 & 2.10 & 2.07 & 2.08 & 2.14 & 2.06 & 2.02 & 2.16 \\
\hline " & 2.16 & 2.16 & 2.19 & 2.04 & 2.02 & 2.11 & 2.14 & 2.08 & 2.04 & 2.14 \\
\hline
\end{tabular}

Table 1. Shows the results of 'S' for different photon numbers of ten sequences trials.

Where:

$\mathrm{n}$ : number of photon pairs 


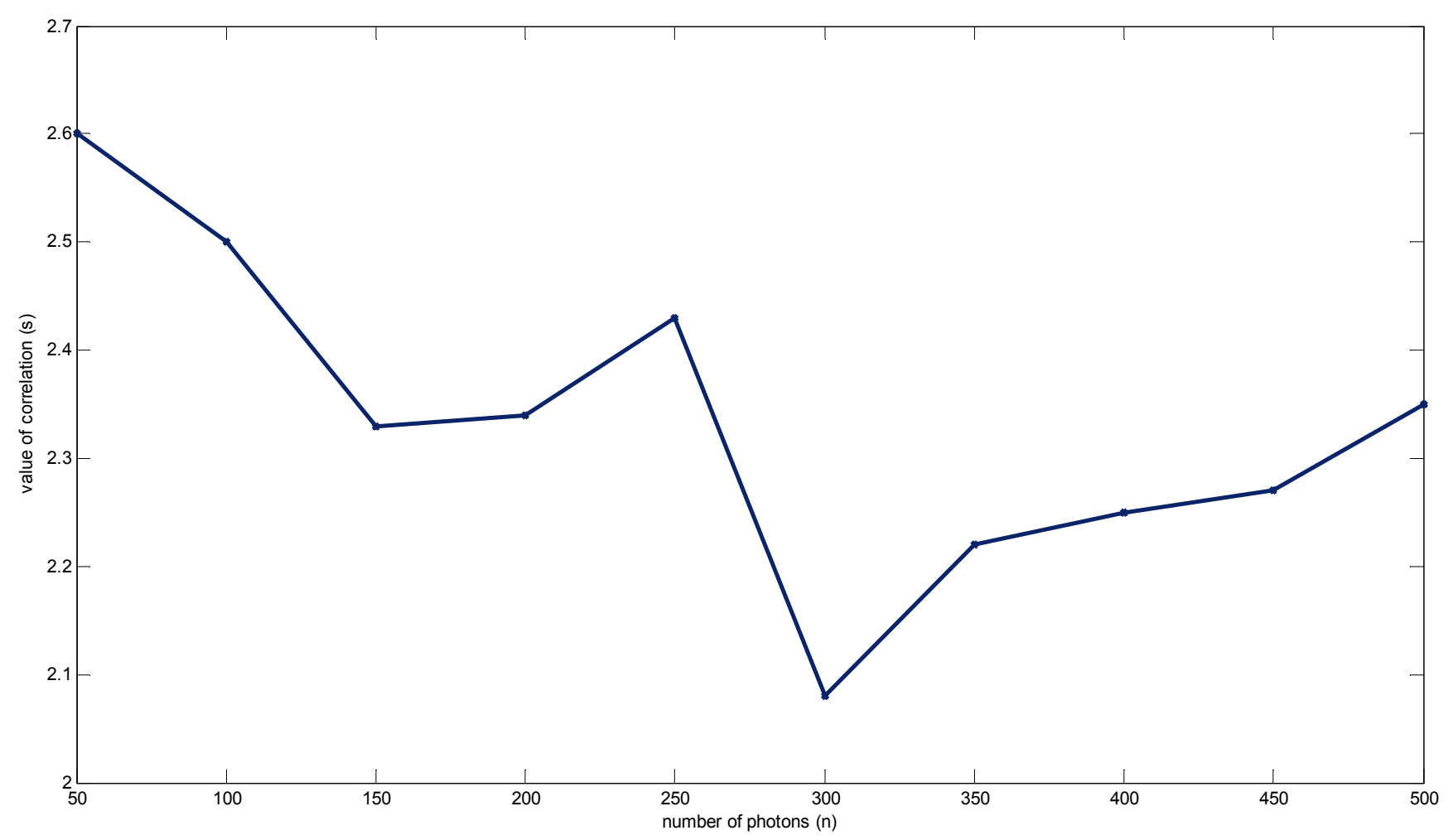

Figure 3. Correlation (S) vs number of photons (n).

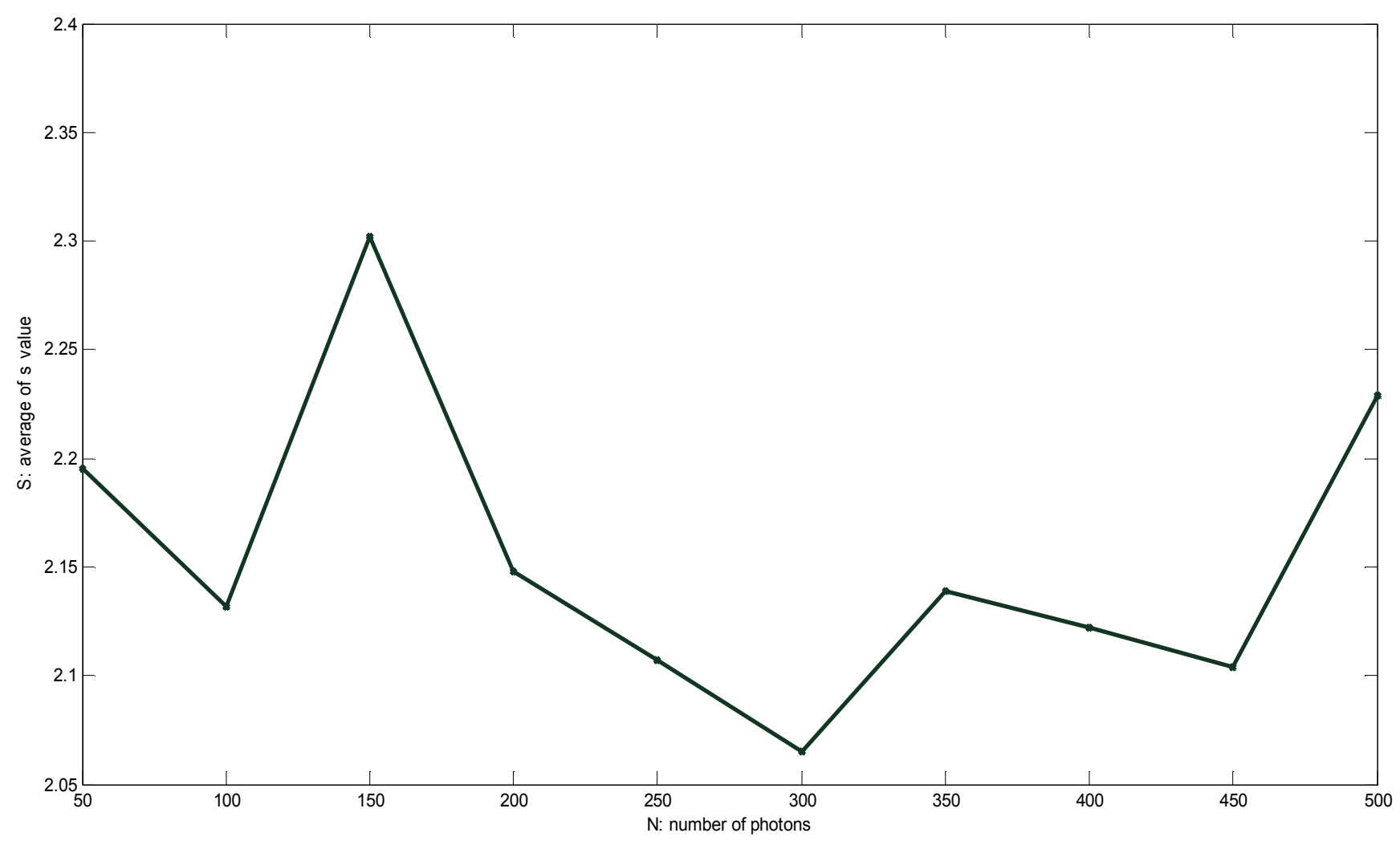

Figure 4. Average correlation vs number of photons. 
CHSH Bell's inequality for local hidden variables theory predicts that

$-2 \geq \mathrm{S}_{\mathrm{LHV}} \leq 2$, and for Quantum mechanics CHSH predicts $\mathrm{S}_{\mathrm{QM}}=2.83$. By this comparison any result's value above "2" will recognized as a violation of classical correlation $\left(\mathrm{S}_{\mathrm{LHV}}\right)$. The results showed an obvious violation but the values of ' $S$ ' were not stable.

When the number of photon pairs is little there is disturbance on the ' $\mathrm{S}$ ' value and that disturbance decreases with increasing of $n$, the largest value of ' $S$ ' was obtained when $\mathrm{n}$ in the range of 50 and 250 as shown in the results presented in the table1, also it was noticed that the 300 value of $\mathrm{n}$ is not suitable to show violation of classical correlation since it gave the least values among all other results. The values of ' $\mathrm{S}$ ' when $\mathrm{n}$ large than 300 tend to become stable at values above ' 2 '. This because that the large number enables all measurement options to take place.

Also there is other reason of disturbance, when the incidence angle is $45^{\circ}$ the result must be randomly, because the results of ' +1 ' and ' -1 ' had the same probability. This randomize will differ from trial to other and by just comparing between ' $\sin ^{21}$ and ' $\cos ^{2}$ one cannot simulate the exact naturally quantum probability measurement results of polarizing beam spitter.

\section{Conclusion}

In this work the trial of testing the quantum correlation by using semiclassical program model was established by representing the probability amplitude of single photon emerged from BPS just as Malus intensity law. And by treating the entangled pairs measurement independently the model calculated the CHSH Bell's inequality. The result showed violation of classic constrains. So by this argument one can get results of CHSH Bell's inequality have unstable value above ' 2 '. But it is not useful method to make simulation program of Ekert's protocol using this method of (PBS), because Ekert's protocol needs constant value of $\mathrm{S}$ to detect the eavesdropper. And the representing of polarizing beam splitter by just comparing $\left(\cos ^{2}\right)$ with $\left(\sin ^{2}\right)$ is not sufficient to give exact quantum prediction results.

\section{References}

[1] Graham Jensen, Christopher Marsh and Samantha (2013), Entangled Photons and Bell's Inequality, University of Rochester, downloaded from:http://www2.optics.rochester.edu/workgroups/lukishova/ QuantumOpticsLab/2013/OPT_253/GroupC_Lab1_Sam.pdf, 22/8/2018.

[2] Lorenzo - Maccone (2013), A simple proof of Bell's inequality, Quantum Physics, Am. J. Phys. 81, 85, [doi: $10.1119 / 1.4823600]$

[3] Sergey A. Rashkovskiy (2017), What does a violation of the Bell's inequality prove?, [arXiv:1701.03700 [physics.gen-ph], pp. 23.

[4] Graham Jensen and Samantha, Single and Entangled Photon Sources, University of Rochester, Rochester NY 14627.

[5] Mathwork.com, http//mathwork.com, visited 18/8/2018.

[6] Guillaume Adenier, Noboru Watanabe, and Andrei Khrennikov (2010) A Fair Sampling Test for Ekert Protocol, [DOI: 10.1142/9789814343763_0031].

[7] Danny Laghi (2013), Prof. Gianluca Grignani Anno EPR Paradox and Bell's Theorem30 September 2013.

[8] Muhammad Sadiq (2016), Experiments with Entangled Photons: Bell Inequalities, Non-local Games and Bound Entanglement, Stockholm University, ISBN:978-91-7649-358-8.

[9] Niclas Hoglund and Olaf Jacobson (2013) : Bell's Theorem and Inequalities, with Experimental Considerations, SA104X Degree Project in Engineering Physics, Theoretical Physics Royal Institute of Technology (KTH)Supervisor: Patrik Henelius May 20, 2013.

[10] Joost Hoogeveen, Szczepan Kowalczyk, and Maarten van (2004), An Historical and Modern View on Bell's Inequality, University OF Amsterdam downloaded from: http://www.science.uva.nl/student/scoop/artikels/maart2005/pr bellRC3.pdf, 29/7/2018.

[11] Sara Idris Babiker Mustafa (2007), simulation of quantum cryptography based on Ekert protocol, (PhD thesis: Institute of laser. Sudan University of science and technology), Sudan. 